Trans

continentales

\title{
Transcontinentales
}

Sociétés, idéologies, système mondial

\section{Les camps palestiniens du Liban}

La Syrie, le Hezbollah et le nouveau pouvoir libanais face aux attentes internationales

The Palestinian camps in Lebanon. Syria, the Hezbollah and the new Lebanese Government Confronted to International Expectations

\section{Bernard Rougier}

\section{(2) OpenEdition}

Journals

Édition électronique

URL : http://journals.openedition.org/transcontinentales/272

DOI : 10.4000/transcontinentales.272

ISBN : 978-2-8218-1410-3

ISSN : 1775-397X

Éditeur

Editions de la maison des sciences de l'homme

\section{Édition imprimée}

Date de publication : 31 décembre 2005

Pagination : 51-67

ISBN : 2200-92168-3

ISSN : 1950-1684

\section{Référence électronique}

Bernard Rougier, « Les camps palestiniens du Liban », Transcontinentales [En ligne], 1 | 2005,

document 4, mis en ligne le 03 février 2011, consulté le 08 septembre 2020. URL : http://

journals.openedition.org/transcontinentales/272 ; DOI : https://doi.org/10.4000/transcontinentales. 272

Ce document a été généré automatiquement le 8 septembre 2020.

Tous droits réservés 


\title{
Les camps palestiniens du Liban
}

\author{
La Syrie, le Hezbollah et le nouveau pouvoir libanais face aux attentes \\ internationales \\ The Palestinian camps in Lebanon. Syria, the Hezbollah and the new Lebanese \\ Government Confronted to International Expectations
}

\section{Bernard Rougier}

À la mémoire de Samir Kassir, journaliste et historien libanais assassiné à Beyrouth le 2 juin 2005.

Depuis le mois de septembre 2004, le Liban a été le théâtre d'une lutte d'influence entre la communauté internationale d'une part et le régime syrien d'autre part, dont l'enjeu n'est rien moins que le démantèlement d'un ordre politique et militaire patiemment édifié par Damas et ses alliés libanais depuis 1990, après quinze années d'un conflit complexe dont on doit rappeler les multiples imbrications.

Guerre gigogne, mêlant perpétuellement dynamiques régionales et dynamiques locales, la guerre du Liban (1975-1990) avait été, successivement ou cumulativement, une guerre civile (entre milices chrétiennes et l'alliance Organisation de libération de la Palestine/gauche libanaise de 1975 à 1977, puis entre milices musulmanes prosyriennes et milices chrétiennes, alliées à Israël de 1978 à la fin des années 1980); une guerre régionale arabe (entre la Syrie et l'OLP, de 1976 à 1990) ; une guerre israéloarabe et israélo-palestinienne (lors de la double invasion israélienne de 1978 au Sud et de 1982 jusqu'à Beyrouth); une guerre intra-communautaire (entre milices chi'ites Amal et Hezbollah en 1988-1989, entre l'armée du général Aoun et la milice chrétienne des Forces libanaises en 1990-1991); une guerre libano-palestinienne (entre milices chrétiennes et OLP de 1975 à 1978, avec un soutien syrien aux premières en 1976, puis entre l'OLP et la milice Amal de 1985 à 1987) ; une guerre libano-syrienne (entre milices libanaises pro-palestiniennes et armée syrienne en 1976-1977, puis entre les milices chrétiennes et l'armée syrienne en 1978, enfin entre les armées libanaise et syrienne en 1989-1990, après que le général Michel Aoun, alors commandant en chef de l'armée et premier ministre libanais par intérim, eut déclaré une « guerre de libération » contre la 
Syrie). Ce cycle de guerres libanaises s'est achevé avec la mise en place d'une hégémonie syrienne complète le 13 octobre 1990, lorsque Damas obtint un feu vert américain pour éliminer militairement le général Aoun, déjà marginalisé par son opposition aux accords de Taëf, et bientôt contraint à l'exil. Seul le Hezbollah put continuer à mener une guérilla armée contre l'occupation israélienne au Sud-Liban (1990-2000).

3 Votée le 2 septembre 2004 par le Conseil de sécurité de l'ONU à l'initiative de la France et des États-Unis, la résolution 1559 reprenait les principales dispositions des accords de Taëf en leur donnant une nouvelle légitimité internationale, plus de quinze après leur signatur ${ }^{1}$. La résolution internationale demandait notamment, dans une allusion transparente à la présence de l'armée syrienne sur le territoire libanais, le retrait du Liban «de toutes les forces étrangères qui y sont encore » - retrait qui, selon Taëf, aurait dû être effectif en 1992, deux après l'introduction des réformes dans la Constitution.

4 De même, en exigeant le "désarmement et à la dissolution de toutes les milices libanaises et non libanaises ", conformément aux textes de Taëf, la résolution de l'ONU appelait au rétablissement de l'autorité de l'État au Sud-Liban et dans les camps palestiniens, détruisant au passage le discours légitimatoire du Hezbollah, selon lequel l'organisation chi'ite aurait cessé de rentrer dans cette catégorie en raison d'une résistance militaire exclusivement dirigée contre l'occupation israélienne dès le début des années 1990.

5 Le volet interne de la résolution tentait, quant à lui, de rétablir le principe de la séparation des pouvoirs, à la fois dans sa définition classique et dans sa dimension syrolibanaise, en rappelant que le respect du calendrier constitutionnel libanais passait par la tenue de nouvelles élections présidentielles «en dehors de toute interférence ou influence étrangère ». Au lieu de quoi, le président syrien Bachar al-Assad préféra, deux jours après la décision du Conseil de sécurité, prolonger le mandat du président Émile Lahoud pour trois années supplémentaires. Au-delà de la question de la reconduction du président, la résolution 1559 cherchait à mettre un terme à l'intrusion de plus en plus pesante des services de sécurité dans la vie politique libanaise, notamment depuis l'arrivée du général Lahoud à la présidence de la République en novembre $1998^{2}$.

6 Acculé à la démission, l'ancien premier ministre Rafic al-Hariri était devenu, à quelques mois des élections législatives, le principal opposant du président et de ses alliés du Hezbollah lorsqu'il fut assassiné le 14 février 2005 dans la capitale libanaise. Dès lors, la pression de la communauté internationale, conjuguée avec celle d'une partie considérable de l'opinion libanaise telle qu'elle s'était exprimée lors du rassemblement du 14 mars sur le lieu symbolique de la place des Martyrs au cœur de Beyrouth, entraîna la création d'une commission d'enquête de l'ONU sur l'assassinat de Hariri et obligea l'armée syrienne à retirer ses troupes du Liban le 26 avril 2005, vingt neuf ans après son entrée dans le pays le $1^{\text {er }}$ juin 1976. Pour la première fois, les aspirations d'une partie majoritaire de la société libanaise ont été prises en compte et soutenues par un système international longtemps indulgent par rapport aux intérêts syriens au Liban.

7 Cependant, la dynamique du changement s'est heurtée à la réalité du système confessionnel libanais et de ses pièges ainsi qu'à la résistance des alliés politiques de la Syrie. Ces derniers ont réussi, parfois avec l'aide involontaire de leurs adversaires, à empêcher la manifestation du 14 mars de se transformer en un mouvement national 
transcommunautaire. Les milieux proches de la présidence ont d'abord usé des ressources mises à leur disposition par le système électoral libanais, en maintenant une loi électorale propre à la réactivation des clivages confessionnels, et que l'opposition plurielle libanaise n'a pas su, ou pas voulu, amender. Dans la période post-électorale, ils ont habilement exploité les déceptions locales provoquées par la victoire électorale du clan Hariri lors des élections de l'été 2005 - victoire disproportionnée par rapport au poids réel de la famille dans la sociologie électorale libanaise. Parallèlement à cette série de coups tactiques, les dirigeants du Hezbollah ont récupéré l'initiative politique, en dramatisant la question du désarmement de leur organisation, présentée comme un enjeu existentiel pour la sécurité du pays et la préservation de la paix civile. Enfin, les assassinats ciblés contre des personnalités qui ont animé la manifestation du 14 mars ont tétanisé les élus de l'ancienne opposition, réinstallant dans le discours public des précautions de langage sur le rôle de la Syrie au Liban, qui avaient progressivement disparu.

8 À travers l'enjeu du désarmement des camps, cet article se propose de montrer comment le régime syrien et ses alliés libanais ont mobilisé des soutiens en milieu palestinien pour faire obstacle à l'application de la résolution 1559 et la façon dont les acteurs politiques et religieux ont réagi à ces sollicitations. Entrée privilégiée pour étudier l'établissement d'un mode de gouvernement reposant essentiellement sur les services de sécurité syro-libanais depuis 1990, la question des camps palestiniens au Liban témoigne aujourd'hui, depuis le retrait de l'armée syrienne, d'une implication de plus en plus lourde du Hezbollah dans la gestion des expressions politiques palestiniennes. Elle illustre à cet égard le rôle tenu par les acteurs religieux dans la mise en place d'un mécanisme de contrôle politique particulièrement aliénant pour les réfugiés eux-mêmes. Les mobilisations provoquées par la résolution 1559 permettent ainsi de considérer les camps comme une arène au sein de laquelle se rencontrent, s'allient ou s'affrontent, au gré des circonstances, des acteurs étatiques (régime syrien), para-étatiques (Hezbollah libanais, Autorité palestinienne) ou transnationaux (réseaux jihâdistes).

\section{Les ressorts politiques d'une exclusion sociale et géographique}

9 Il existe sur le territoire libanais une dizaine de camps de réfugiés palestiniens, où vivent plus de la moitié des 399152 réfugiés officiellement enregistrés au Liban par l'agence des Nations unies pour les réfugiés palestiniens ${ }^{3}$. La génération qui a quitté la Galilée en 1948 - aujourd'hui moins de $5 \%$ de cette population - est en voie d'épuisement démographique, celle qui lui a succédé a connu la naissance de l'Organisation de libération de la Palestine (OLP) et s'est engagée dans l'action armée, tandis que les générations suivantes (troisième, voire quatrième génération) vivent dans le désenchantement idéologique et l'exclusion sociale ${ }^{4}$.

En effet, depuis la fin de la guerre (1990), un dispositif exceptionnel a été conçu pour maintenir les réfugiés aux marges de la société libanaise ${ }^{5}$. Les responsables libanais justifient leur refus de toute mesure d'intégration - de nature sociale, juridique, ou professionnelle - par l'idée que de telles dispositions seraient interprétées, à la fois par les intéressés et par la communauté internationale, comme l'acceptation tacite de leur implantation (tawtîn) définitive au Liban. Si elle était mise en œuvre, une telle 
perspective, expressément rejetée par les accords de Taëf, bouleverserait les équilibres communautaires libanais en conduisant à la naturalisation de plusieurs milliers de réfugiés de confession sunnite.

11 Cette préoccupation interne recoupe par ailleurs une préoccupation régionale plus fondamentale encore, puisque l'avenir des Palestiniens du Liban est lié au règlement du conflit israélo-palestinien et à la question sensible du droit au retour des réfugiés «dans leur foyer d'origine $»^{6}$. Dès lors, les enjeux posés par la question palestinienne au Liban - aussi bien la définition de la politique de l'État à l'égard des réfugiés que l'évolution des forces politiques et militaires à l'intérieur des camps - engagent directement les intérêts vitaux de la Syrie dans son espace régional. Dès leur entrée au Liban, les Syriens ont cherché à domestiquer le facteur palestinien, à la fois pour en faire un instrument de politique étrangère et empêcher que l'affirmation nationale palestinienne ne se fasse à leur détriment.

12 Fortement politisés dès la fin des années 1960, les camps du Liban ont constitué les bases naturelles de l'OLP pendant la guerre. Entraînés par leur alliance avec une opposition libanaise hétéroclite, les Palestiniens étaient sur le point de l'emporter sur leurs adversaires phalangistes lorsque Hafez al-Assad fit intervenir son armée au Liban en juin 1976, prenant prétexte des risques induits par la mise en place d'un pouvoir révolutionnaire "palestino-progressite » pour obtenir l'assentiment des États-Unis (et implicitement d'Israël) à sa politique libanaise. Prise entre l'étau syrien et les frappes israéliennes, l'OLP dut subir dans les années 1980 l'hostilité de la communauté chi'ite en voie de militarisation. Après que son infrastructure militaire eut été détruite par l'invasion israélienne de 1982, la centrale palestinienne, qui avait, entre-temps, fait revenir des combattants au Liban, fit l'objet « d'une guerre des camps » de la part de la milice pro-syrienne Amal, sous la forme d'un siège de deux ans des camps de Beyrouth et du Sud (1985-1987), ponctuée par de violentes phases de bombardements ${ }^{7}$.

13 Avec la fin de la guerre, les services de sécurité syriens ont continué à combattre l'influence politique de Yasser Arafat et à détruire les restes du cadre institutionnel palestinien symbolisé par l'OLP. Dans les camps qui n'étaient pas encore soumis à l' imperium syrien, autour de Beyrouth et de Saïda, tous les lieux de pouvoir (comités populaires, postes de responsabilité au sein de l'UNRWA) ont été investis par les factions dissidentes palestiniennes (Front populaire de libération de la Palestine (FPLP)Commandement général, Saïka, Fatah-Intifada) organiquement reliées au régime syrien. Ces organisations étaient en mesure de faciliter l'obtention d'un passe-droit ou l'expression d'une requête grâce à leur accès à l'officier syrien - à Saïda par exemple, ce rôle a longtemps été dévolu à celui que les Palestiniens appelaient le général Nassâr.

En récompensant de la sorte ses alliés palestiniens, le régime poursuivait sa guerre contre le principe de «l'indépendance de décision palestinienne » dont Arafat était le symbole honni. La seule existence d'un acteur palestinien indépendant empêchait la Syrie de détenir à elle seule les clefs d'un règlement régional avec Israël et d'imposer son hégémonie sur l'ensemble des acteurs de l'Orient arabe. Après la conclusion des accords d'Oslo en 1993, la chasse aux militants du Fatah s'intensifia au Liban, tandis que les alliés politiques de la Syrie manifestaient au quotidien leur détestation du président de l'OLP, unanimement accusé dans les médias libanais de sacrifier les exigences du droit au retour pour prix de sa reconnaissance par le gouvernement israélien. Pendant toute la durée du processus d'Oslo, les membres du Fatah ont d'ailleurs été considérés par les Syriens et leurs alliés libanais comme des supplétifs d'Israël, qu'il fallait 
combattre et éliminer dans l'espace libanais, grâce à une palette de moyens allant de la condamnation judiciaire à l'assassinat physique - pratique dont furent victimes plusieurs dizaines de cadres palestiniens dans le Liban d'après-guerre.

La lutte contre le Fatah d'Arafat comprenait un prolongement domestique non moins important. L'organisation palestinienne avait en effet vécu en osmose avec les secteurs musulmans de la société libanaise au début des années 1970 et les responsables syriens avaient compris, à juste titre, que les principales menaces pour leurs intérêts surgiraient de ce milieu, en particulier dans la communauté sunnite. En écho aux affrontements des premières années de la guerre du Liban, pendant lesquelles l'armée syrienne avait combattu la gauche libanaise pro-palestinienne, des solidarités historiques pouvaient être réactivées à la faveur d'un nouveau contexte. Par la suite, l'aide apportée par Yasser Arafat aux Frères musulmans syriens en lutte contre le régime de Hafez al-Assad de 1978 à 1982 illustrait la continuité de la menace, en dépit du changement de registre idéologique. Face au resserrement de l'étau syrien au Liban, intellectuels progressistes, activistes islamistes ou militants de l'indépendance palestinienne pouvaient trouver dans les camps palestiniens l'expérience et la main d'œuvre combattante indispensable à leur survie politique. Dès leur retour à Beyrouth en 1987, les Syriens veillèrent d'ailleurs à réduire au silence toutes les oppositions à leur tutelle en provenance des milieux sunnites libanais, laissant au besoin la milice chi'ite Amal accomplir les œuvres de basse police. Dans la continuité de cette politique, Amal fut encouragée par la direction militaire syrienne à bombarder les camps de réfugiés de Beyrouth pendant l'épisode dramatique de la "guerre des camps" (1985-1987). Depuis son établissement en 1970 suite au coup d'État de Hafez al-Assad, le régime syrien a cloisonné et encadré les expressions politiques d'une population qui, par son origine confessionnelle sunnite, son enracinement à proximité des grands centres urbains, ses liens familiaux élargis à toute la région et son attachement politique à la cause palestinienne, illustrait le mieux la continuité humaine et géographie de la "Grande Syrie » (bilâd al-châm) - ensemble géographique qui a, par ailleurs, servi à justifier les prétentions régionales de la famille Assad.

\section{La question palestinienne au cœur du dispositif sécuritaire libano-syrien}

Comme tant d'autres enjeux qui avaient trait à la pérennité des intérêts du régime syrien au Liban, l'étude des modes de gestion de la question palestinienne permet de mieux comprendre comment les institutions politiques ont été vidées de leur contenu et remplacées par des processus de décision à la fois opaques dans leur fonctionnement, et transparents dans leurs résultats. Tout au long des années 1990, l'autorité politique de l'État libanais a été minée de l'intérieur par la constitution d'un noyau sécuritaire sous contrôle syrien s'attribuant la capacité de définir des domaines réservés. Cette dépossession s'est manifestée au sommet de l'État : ainsi, le conseil des ministres - instance collégiale et incarnation du pouvoir exécutif selon les accords de Taëf - n'a jamais pu discuter de la question palestinienne au Liban, en raison des enjeux nationaux et régionaux dont elle était le catalyseur. Pendant les quinze années de la tutelle syrienne au Liban, les différents ministères de l'État libanais, comme celui de l'Intérieur ou des Affaires étrangères, n'ont joué aucun rôle dans la définition d'une politique vis-à-vis des réfugiés palestiniens au Liban. De même, cette paralysie 
institutionnelle a empêché l'élaboration d'une réflexion sur les perspectives sociales et politiques offertes aux réfugiés, privant l'État d'une expertise précieuse pour préparer l'avenir et abandonnant cette fonction à des centres de recherches étrangers ${ }^{8}$. À l'exception du règlement de questions administratives liées au déplacement des personnes, le rôle du gouvernement libanais s'arrêtait dès l'instant où se manifestait l'intérêt des services de sécurité syriens au Liban. Dans ce cadre, la compétence des principales agences de sécurité libanaises - comme la Sûreté générale, sous l'autorité de son directeur Jamil al-Sayyid, ou les services de renseignements de l'armée, se limitaient à l'accomplissement de tâches de sous-traitance, en coordination avec l'autorité syrienne du moment. À l'époque (1990-2000) où le général syrien Ghazi Kana'an assurait la direction de ces services depuis le village d'Anjar dans la Bekaa libanaise, c'est son adjoint Rustom Ghazalé qui, depuis le siège de l'hôtel Beau Rivage à Beyrouth, définissait des objectifs et donnait les instructions que ses différents interlocuteurs palestiniens étaient chargés de diffuser dans les camps. Cette dépossession a été rapidement comprise et intériorisée par les différents acteurs du dossier palestinien. Au total, « les Libanais ne pouvaient rien faire sans l'autorisation préalable des Syriens » comme le dira une personnalité importante dans le dispositif de liaison palestino-syrien.

La préoccupation politique essentielle des Syriens était de réduire par tous les moyens la marge d'influence du Fatah afin d'empêcher Yasser Arafat de reprendre le contrôle des camps et de parler au nom des réfugiés palestiniens du Liban. En décembre 1999, le procureur général 'Adnan 'Addoum avait été à l'origine de la condamnation à mort par contumace du représentant de l'Autorité palestinienne au Liban, Sultan Abou al-'Aynân, pour « formation d'une bande armée ». Prise sur l'initiative de celui qui était alors le chef des renseignements syriens au Liban, le général Ghazi Kana'an, la décision de justice manifestait la volonté syrienne de retirer à Yasser Arafat le droit de parler au nom des réfugiés palestiniens du Liban lors de négociations futures avec Israël, tant que la Syrie ne serait pas elle-même associée à de telles négociations. Elle sanctionnait ainsi une initiative diplomatique de l'Autorité palestinienne, car celle-ci avait utilisé à cette occasion les canaux institutionnels pour expliquer aux responsables libanais les positions défendues par la délégation palestinienne à l'approche des négociations israélo-palestiniennes qui devaient avoir lieu à Camp David pendant l'été 2000. À un second niveau, la condamnation adressait aussi un message à l'ensemble du personnel politique libanais, à qui il était signifié l'interdiction de rentrer en contact avec Yasser Arafat sur une question aussi sensible pour les intérêts syriens.

On doit noter que cette politique syrienne n'a jamais été assumée comme telle. Les Syriens ont fait endosser par des acteurs libanais l'ensemble des mesures législatives, judiciaires et militaires qui ont exclu les Palestiniens de la reconstruction libanaise. Le refus de l'implantation définitive des palestiniens du Liban (tawtîn) a servi de prétexte pour justifier l'alliance entre les chrétiens pro-syriens et la Syrie. À plusieurs reprises, le président Lahoud a d'ailleurs justifié la prorogation inconstitutionnelle de son mandat en septembre 2004 par la nécessité impérieuse de lutter contre l'implantation palestinienne - sans jamais dénoncer les conditions peu transparentes d'attribution de la nationalité libanaise à différents clients proches de la Syrie, d'origine syrienne, kurde ou palestinienne. Lors de sa nomination à la présidence de la République libanaise en novembre 1998, il avait fait de ce thème l'une des raisons d'être de son mandat, parvenant même à réussir la synthèse populiste entre refus du tawtîn et lutte contre la 
corruption, en prêtant à son adversaire Rafic al-Hariri la volonté d'implanter les réfugiés moyennant versement de compensations financières par les institutions internationales.

\section{La riposte syrienne à la résolution 1559}

Peu après le vote de la résolution 1559 par le Conseil de sécurité en septembre 2004, le pouvoir syrien a exploité les dividendes d'une politique de division et de contrôle de l'ensemble des camps palestiniens pour mettre au point sa riposte politique.

Les relais de l'influence syrienne en milieu palestinien ont été mis à contribution pour empêcher toute velléité de coopération entre acteurs palestiniens et gouvernement libanais en vue d'un possible désarmement des camps palestiniens.

21 Le 12 février 2005 - deux jours avant l'assassinat de Rafic al-Hariri -, le représentant officiel de l'Autorité palestinienne au Liban, Sultan Abou al-'Aynân, avait été convié à se rendre à Damas à la demande du vice-ministre des Affaires étrangères, Walid alMu'allim, désormais en charge du dossier libanais. Sous le prétexte d'un examen d'ensemble de la situation des Palestiniens au Liban, les Syriens ont obtenu de leurs interlocuteurs une condamnation de la résolution 1559 et l'engagement de coordonner leur position avec celle du Hezbollah. Avec un certain cynisme, ils ont d'ailleurs fait mine de découvrir, lors des discussions tenues dans la capitale syrienne, les souffrances des réfugiés palestiniens au Liban, en promettant à la délégation palestinienne de faire ultérieurement pression sur le gouvernement libanais pour obtenir un allégement des contraintes pesant sur cette population'.

Il est intéressant de connaître l'identité de ceux qui sont aujourd'hui chargés de mettre en place cette politique de coordination, dont le but est d'illustrer, à un moment critique de l'évolution des rapports libano-syriens au Liban, « le caractère inséparable » des volets palestiniens, libanais et syriens sur la question de la (non) application de la résolution 1559. Deux acteurs essentiels du réseau syrien en milieu palestinien ont discrètement mis au point les modalités de la rencontre de Damas: Oussama 'Abbas et Jamâl Sleïmân. Seul palestinien à avoir appartenu au Bureau politique de la Jama'a islâmiyya libanaise avant de présenter sa démission en 1998, ingénieur de formation, ayant accès aux milieux jihâdistes de Aïn el-Héloué grâce à son passé de combattant dans les rangs de la Jama'a lors des combats menés contre les Forces libanaises à l'est de Saïda en 1985, Oussama 'Abbas a été l'un des principaux interlocuteurs des officiers syriens en charge du dossier palestinien, Rustom Ghazalé, puis Jami'a. Chargé des renseignements auprès du Hezbollah et des services syriens, son ami Jamâl sleïmân est un ancien militant du Fatah. Passé au Hezbollah dans la seconde partie des années 1980, il dispose d'une milice dans le camp de Mié-Mié après avoir été chassé du camp de Aïn el-Héloué à la fin des années 1980 par des militants du Fatah loyaliste. Enfin, Jamal Khalil, qui accompagnait la délégation palestinienne à Damas, est le principal relais syrien dans le camp de Rashidiyyé, où Sultan Abou al-'Aynân est assigné à résidence depuis sa condamnation à mort.

L'invitation faite à Sultan Abou al-'Aynân était en soi la manifestation du caractère instrumental que revêtaient les institutions judiciaires libanaises pour le pouvoir syrien. Condamné à mort par contumace pour "formation de bande armée " en décembre 1999, au moment où la Syrie était soucieuse de limiter au maximum les capacités d'influence de Yasser Arafat sur la scène libanaise, Sultan Abou al-'Aynân 
était, cette fois-ci, traité avec tous les égards dus à un allié potentiel de Damas et autorisé à franchir la frontière libano-syrienne sous l'escorte de l'armée libanaise grâce à l'intervention expresse de ceux-là même qui avaient été à l'origine de sa condamnation pénale six années auparavant.

La mise en place d'un canal de communication avec Sultan Abou al-'Aynân était aussi un moyen pour les responsables syriens de court-circuiter l'autorité du nouveau président de l'Autorité palestinienne, Mahmoud 'Abbas, élu peu après le décès de Yasser Arafat. Il s'agissait pour Damas de récupérer l'allégeance politique des cadres du Fatah au Liban, dans un contexte de crise générale des institutions palestiniennes et de graves dissensions internes dans les territoires palestiniens (ré)-occupés, y compris au sein du Fatah. Les déclarations des responsables du Fatah au Liban sont toutes allées dans le sens de l'interprétation syrienne, pointant ici une application sélective des résolutions de l'ONU oublieuse du droit au retour (résolution 194) et de l'obligation pour l'armée israélienne de se retirer des territoires occupés en 1967 (résolution 242), accusant là une « décision israélo-américaine visant à priver la résistance des moyens de faire face à l'occupation israélienne $»^{10}$ ou refusant encore que les groupes palestiniens armés ne rentrent dans la catégorie de «milices». Tombant à leur tour dans le piège de l'approche globale prônée par la Syrie, les responsables libanais du Fatah ont considéré que le désarmement des camps était une question politique plutôt que militaire, qui devait à ce titre être réglée lors de négociations régionales mettant à plat l'ensemble des questions en suspens. Les effets de ce rapprochement ont été visibles à Aïn el-Héloué dans la banlieue est de Saïda au Sud-Liban, où une structure de coordination provisoire a été montée en mai 2005 pour intégrer des factions fidèles à la Syrie comme le FPLP-Commandement général, la Saïka, le Hamas, le Jihad islamique, avec des organisations de l'OLP comme le Fatah, le FPLP et le FDLP (Front démocratique de libération de la Palestine). Un « comité de suivi » pour gérer les affaires intérieures du camp a aussi été créé. Après une posture souvent héroïque pendant les quinze années de tutelle syrienne sur le Liban, les responsables du Fatah ont pris le risque, au nom de calculs à court terme, de rompre avec une indépendance de décision qui leur avait permis de résister aux pressions de leur environnement politique immédiat. Quant à ceux des militants de base du Fatah qui étaient plutôt favorables à la résolution 1559, ils se sont trouvés mis en minorité par la direction locale du mouvement pendant les discussions internes menées au sein de l'organisation palestinienne après le vote de la résolution.

25 Face à la menace d'un nouvel affaiblissement de son organisation, le président de l'Autorité palestinienne Mahmoud 'Abbas a considéré que les Palestiniens devaient s'abstenir de toute intervention dans les affaires intérieures libanaises. Cette attitude fut endossée le 29 mars 2005 par le Conseil révolutionnaire (al-majlis al-thawri) du Fatah pour lequel l'OLP «n'était pas partie prenante dans ce qui se passait au Liban». Conscient du risque de voir le dossier palestinien exploité sur la scène libanaise, Mahmoud 'Abbas ne se priva pas lors de ses passages à Beyrouth de confier en privé à certains interlocuteurs de l'opposition sa prédisposition à accepter un désarmement des camps du Liban, conformément à la résolution 1559, moyennant l'obtention d'un statut pour les réfugiés et une amélioration de leurs conditions d'existence. Lui-même confronté à la concurrence de pouvoirs rivaux dans la perspective du retrait israélien de Gaza, Mahmoud 'Abbas ne pouvait pas défendre le maintien de zones de non-droit au Liban dans un contexte où, depuis le vote de la résolution par le Conseil de sécurité, la communauté internationale cherche à encourager et à accompagner le 
recouvrement par l'État libanais de sa souveraineté. Un consentement palestinien à la résolution 1559 ne peut toutefois être publiquement affirmé, car il vaudrait immédiatement à son auteur une volée de mises en accusations pour trahison des «constantes» islamo-nationalistes. De nature tactique, la position d'attente du président de l'Autorité palestinienne vise à maintenir l'illusion d'une unité minimale entre les différents acteurs de la scène palestinienne au Liban.

\section{L'intervention du Hezbollah sur la question des camps} incombe principalement au Hezbollah, devenu de facto le dépositaire des intérêts syriens au Liban et le principal animateur de l'opposition à la résolution 1559. Membre depuis une dizaine d'années du bureau politique du Hezbollah, Hassan Hudrouj (Abou Mahmoud) est chargé des contacts avec les groupes palestiniens. Originaire de l'un des « sept villages » situés du côté israélien de la frontière mais revendiqués par le Liban, il présente la particularité d'être à la fois de confession chi'ite et d'origine palestinienne ${ }^{11}$. Son rôle consiste à établir des contacts quotidiens avec tous les acteurs $\mathrm{du}$ spectre palestinien, des organisations dissidentes pro-syrienne comme le FPLPCommandement général jusqu'aux représentants du Fatah loyalistes. Lui-même membre du Fatah jusqu'en 1982, il a rejoint les rangs du Hezbollah juste après l'invasion israélienne. Sa trajectoire est d'ailleurs significative de l'inversion d'une dynamique par laquelle le Fatah avait d'abord formé l'essentiel des cadres militaires et politiques chi'ites à la fin des années 1970 , avant de se retrouver victime de ses créatures dans la seconde moitié des années 1980, lorsque le conflit militaire avec l'organisation chi'ite Amal laissa la place à un conflit politique et idéologique avec le Hezbollah. Conscient de ce que les camps représentent « un maillon faible " par lequel le volet sur le désarmement pourrait connaître une application qui le placerait en ligne de mire de la communauté internationale, le Hezbollah s'est lancé dans une diplomatie visant à dissuader toute expression dissidente en provenance des camps, au nom de l'affirmation d'une solidarité de principe entre l'organisation chi'ite et le peuple palestinien face à la menace d'une agression sioniste. Depuis septembre 2004, un mot d'ordre a été donné, demandant aux factions armées de ne plus vendre des armes à l'extérieur des camps. Au niveau local, le Hezbollah soutient ses alliés pour empêcher le Fatah de reprendre le contrôle des camps après le départ des Syriens, comme il y était parvenu au mois d'avril au camp de Chatila, à l'ouest de Beyrouth, en écartant les éléments pro-syriens du comité populaire (Saïka, FPLP-Commandement général, FatahIntifada). La même opération n'a pas pu se reproduire dans le camp de Bourj al-Barajné, situé dans le prolongement de la banlieue sud, gérée et contrôlée par le Hezbollah, où le Commandement général a gardé le contrôle du comité populaire. L'organisation d'Ahmed Jibril, le FPLP-Commandement général, a d'ailleurs acheminé des armes de la Bekaa dans le camp pour consolider sa présence.

L'influence du Hezbollah s'est aussi exercée au sein du système politique, puisqu'un ministre proche du parti islamiste chi'ite, Trad Hamadé, lui-même ancien responsable politique du Fatah dans la région du Hermel avant l'invasion israélienne de 1982, a pris, le 3 juin 2005, la décision d'assouplir les conditions draconiennes qui interdisaient aux travailleurs palestiniens l'accès au marché de l'emploi ${ }^{12}$. L'initiative, sans modifier l'exclusion de principe qui frappe les Palestiniens au Liban, peut se lire comme une 
mesure visant à encourager les organisations palestiniennes à poursuivre la coopération ouverte par le Hezbollah. A contrario, la plupart des décisions discriminatoires à l'encontre des réfugiés ont été prises lorsque Rafic al-Hariri adversaire politique du Hezbollah de la fin de la guerre jusqu'à son assassinat le 14 février 2005 - était premier ministre, même s'il n'en n'a jamais été à l'origine. Les alliés de la formation islamiste chi'ite dans les camps ne manquent d'ailleurs pas de rappeler cette concomitance, comme pour prévenir toute tentative allant dans le sens de l'établissement d'un lien, de nature politique ou humanitaire, entre les réfugiés palestiniens et la famille Hariri. De la sorte, les Syriens et leur allié du Hezbollah ont écarté la possibilité d'une alliance qui aurait modifié l'équilibre confessionnel entre chi'ites et sunnites au Liban, en redonnant à ces derniers des capacités militaires.

Cette ligne plus solidaire affichée par Trad Hamadé s'inscrit dans la continuité d'une stratégie visant à s'emparer de la symbolique palestinienne pour augmenter le prestige régional du Hezbollah. Dès le début de la seconde Intifada en septembre 2000, alors que les Palestiniens des territoires s'interrogeaient encore sur les modalités, civiles ou militaires, de leur mobilisation après l'échec de Camp David, le Hezbollah avait mis en avant la pertinence du recours à la violence armée, seul moyen selon lui - comme l'avait illustré la « libération » du Sud-Liban de l'occupation israélienne en mai 2000 de récupérer les territoires occupés par Israël. Le caractère trompeur de cette symétrie avait été perçu par les militants du Fatah au Liban - à l'évidence, la Cisjordanie ne revêtait pas, pour les Israéliens, la même valeur stratégique que le Sud-Liban - mais cette représentation fausse finit par s'imposer dans la société politique palestinienne, donnant au gouvernement d'Ariel Sharon le prétexte recherché pour détruire l'essentiel des infrastructures de l'Autorité palestinienne. Pendant toute la durée du processus d'Oslo, les instruments de propagande du Hezbollah avaient mis en balance l'héroïsme de la «résistance islamique » avec la pusillanimité de négociateurs palestiniens, accusés de troquer de minuscules avancées territoriales contre le sacrifice de droits fondamentaux et, pire encore, de faciliter la mise en place de mécanismes de coopération avec les militaires israéliens au détriment du Hamas et du Jihâd islamique, les principaux alliés du Hezbollah dans la société palestinienne. Lors des négociations « finales » de Camp David en 2000, Yasser Arafat a incarné au Liban la figure du traître aux intérêts de la nation arabe et islamique - dont tout à la fois la Syrie et le Hezbollah se voulaient les seuls porte-voix - avant d'être décrit par la diplomatie américaine et les médias occidentaux comme le principal obstacle à la paix. À nouveau pendant l'été 2005, les responsables du Hamas et du Jihâd islamique ont comparé le désengagement unilatéral de Gaza avec le retrait israélien du Sud-Liban, en tirant la conclusion que la violence armée était le seul moyen d'obtenir la libération des territoires occupés. De même, ils ont annoncé que les tirs de roquettes sur Israël obéiraient à la même logique que celle qui préside à l'action du Hezbollah vis-à-vis des fermes occupées de Chebaa, omettant à cette occasion le rôle de l'Autorité palestinienne et laissant indirectement planer le spectre de la guerre civile à Gaza ${ }^{13}$.

29 En important en milieu palestinien le discours de guerre véhiculé par la châne de télévision du Hezbollah Al-Manâr, largement regardée dans l'ensemble de la région, les militants radicaux, d'orientation islamiste ou strictement nationaliste, ont progressivement détruit le capital de sympathie et de solidarité constitué auprès de l'opinion occidentale après la première Intifada de 1987. Un tel alignement eut aussi pour effet de reléguer au second plan les acteurs de la société civile au profit de groupuscules armés dont la prétention à parler au nom d'un ensemble plus large ne 
valait pas brevet de représentation de la société palestinienne. Le recours aux attentats suicides, tiré lui aussi des précédents inaugurés par le Hezbollah contre la Force multinationale en 1983, mais étendu cette fois à des cibles civiles israéliennes, allait accroître l'isolement international des Palestiniens, notamment après les attentats du 11 septembre 2001. En réglant leur comportement sur un acteur extérieur à la scène palestinienne, les responsables palestiniens qui empruntent le langage du Hezbollah prennent le risque de perdre la capacité de définir le sens de leur lutte au profit d'une organisation qui, placée dans une situation toute autre, ne possède ni les mêmes intérêts, ni le même agenda stratégique. Le discours de solidarité avec l'environnement islamique et arabe éloigne ainsi les acteurs palestiniens du système international, alors que celui-ci apparaît comme le seul moyen pour eux d'éviter le face à face inégal avec un État aussi puissant que l'État israélien. Ce dernier point illustre ainsi, en dépit des conflits passés, la profonde unité de destin entre Libanais et Palestiniens, puisque, pour les uns comme pour les autres, l'accès à la souveraineté est directement tributaire du degré d'engagement de la communauté internationale et de l'effet modérateur que celle-ci est capable d'exercer sur des voisins trop voraces.

\section{Les milieux religieux des camps face à la résolution 1559}

L'étude des thèmes de la prédication, tels qu'ils sont donnés à entendre depuis plusieurs années aux habitants de Aïn el-Héloué, permet de mieux comprendre comment les prédicateurs des mosquées qui maillent le camp entretiennent un climat permanent de mobilisation : ils construisent une culture du jihâd qui repose, dans sa dimension régionale, sur la dénonciation des agressions dont l'oumma fait l'objet de la part de l'Occident (relayé sur place par des régimes arabes qui ont signé la paix avec Israël) et, dans sa dimension nationale, sur une critique radicale des valeurs constitutives de l'entité libanaise ${ }^{14}$. Les sermons du vendredi fournissent aux entrepreneurs identitaires que sont devenus les prédicateurs de mosquées l'occasion d'intervenir avec virulence dans le débat politique et culturel libanais, en soulevant les inévitables contradictions qui opposent les valeurs du pacte national, d'une part, à celles de l'islam dans sa version salafiste, d'autre part. Cet argumentaire, souvent élémentaire et caricatural, trouve son inspiration dans l'actualité politique telle qu'elle est présentée par les chaînes de télévision arabes et libanaises, malgré le degré de " corruption morale » de ces dernières. Les prédicateurs assurent ensuite un effort de pédagogie islamiste en direction des fidèles, en fournissant l'explication d'événements extérieurs dont la logique cachée est toujours celle, in fine, de la persécution du « seul vrai islam ». Véritable aubaine pour ces prédicateurs, la résolution 1559 leur donne l'occasion de mêler dans leurs prêches convictions idéologiques et intérêts tactiques, enrobant dans un code islamique et culturel le rejet d'un texte qui, s'il était appliqué, ruinerait les conditions matérielles de leur influence dans la "société des camps" palestiniens.

31 Le discours de dénonciation de la résolution 1559 a été porté à Saïda par le cheikh sunnite Maher Hammoud. Héritier d'une famille prestigieuse de Saïda, petit-fils de l'ancien mufti de la ville, diplômé de la faculté de droit religieux de l'université de Damas, cheikh de la mosquée al-Qods (Jérusalem) à Saïda, Maher Hammoud a été l'un des premiers soutiens du Hezbollah en milieu sunnite dès le début des années 1980. 
Membre constitutif du « Rassemblement des oulémas musulmans » conçu pour rallier les religieux sunnites à la révolution khomeyniste, Maher Hammoud continue d'assumer une fonction d'intermédiaire entre l'appareil sécuritaire syrien au SudLiban, le Hezbollah et les milieux radicaux du camp de Aïn el-Héloué. Quelques jours après le rassemblement des différents courants de l'opposition le 14 mars 2005 place des Martyrs à Beyrouth, le cheikh Hammoud a été invité à s'exprimer sur la chaîne de télévision Al-Risâlat («le Message ») créée à l'intérieur du camp de Aïn el-Héloué par différents groupes jihâdistes et exclusivement captée par les habitants du camp disposant du câble. Lors de l'émission, il a condamné la résolution 1559 et le rôle d'une opposition libanaise "acquise à Israël et aux États-Unis ». Il a de même appelé les habitants du camp à soutenir la résistance de la Syrie face à l'agression occidentale. Cependant, de telles injonctions ne sont pas reçues sans esprit critique par les intéressés. Ces derniers étaient invités à donner leur point de vue, et l'un d'eux fit remarquer " qu'il ne se sentait pas tenu de défendre le régime syrien après les combats de 1976, la chute de Tell al-Za'atar et le blocus provoqué par la guerre des camps $»^{15}$.

Les mêmes milieux religieux ont diffusé un discours de peur existentielle qui exploite volontiers la mémoire des traumatismes de la guerre. La libération de Samir Geagea, l'ancien leader de la milice chrétienne des Forces libanaises, a fourni aux prédicateurs l'occasion d'évoquer les massacres de Sabra et Chatila pour justifier le refus de désarmer les camps, à l'horizon de nouvelles menaces venues des régions chrétiennes. De même, le retrait de l'armée syrienne du Liban, opéré le 26 avril 2005, a été présenté par divers relais de l'influence syrienne comme la perte d'un bras protecteur pour les réfugiés palestiniens, désormais seuls face à l'État et à l'armée libanaise - n'était-ce le soutien apporté par le Hezbollah. Dans le principal camp du Nord-Liban, à Nahr alBâred, un cheikh salafiste a déclaré à ses ouailles, lors d'un prêche prononcé à la fin du mois de mars, « qu'il fallait manifester une solidarité avec le régime syrien, malgré tout ce que nous avons subi de sa part, au nom de la défense de l'islam». Selon lui, l'assassinat de Rafic al-Hariri profite à l'évidence aux États-Unis, et les pressions en faveur d'une application de la résolution 1559 s'inscrivent dans une stratégie d'encerclement de la Syrie, pour empêcher le départ des moujahidîn en Irak ${ }^{16}$.

La prédisposition des milieux radicaux à manifester une solidarité de façade avec un régime syrien dont ils rejettent par ailleurs le caractère impie s'explique par une volonté de conserver un statu quo qui, depuis plus de quinze ans, leur a assuré les moyens de convaincre une partie de la jeunesse et de préparer de nouvelles recrues pour d'autres combats. Les idéologues jihâdistes dénoncent à longueur de prêches et de tracts l'ordre politique régional, la nature impie des États qui le composent et le rôle des États-Unis dans leur survie, mais veillent parallèlement à maintenir en l'état la situation dans les camps. Ils contribuent à détruire la croyance en la légitimité des institutions palestiniennes, fournissent au passage un encouragement éventuel à la suppression de tel ou tel cadre du Fatah, mais s'arrêtent devant la perspective d'un conflit armé. À Aïn el-Héloué, lors de chaque crise interne, le cheikh Jamal Khattâb, prédicateur de la mosquée al-Nour dans le quartier dit de safourriya, assure une fonction de médiation entre les diverses forces politiques du camp. L'art de Jamal Khattâb consiste à tenir une prédication de combat contre l'Occident, les régimes arabes, l'État libanais, l'OLP, les « minorités haineuses » (chrétiens et chi'ites), tout en faisant valoir son autorité morale auprès des plus radicaux - réunis dans la milice salafiste de la "Ligue des Partisans " ('Usbat al-Ansâr) - pour empêcher que le camp ne bascule dans une guerre interne entre islamistes jihâdistes et militants du Fatah qui 
pourrait provoquer l'intervention d'éléments extérieurs, surtout si les islamistes en sortaient vainqueurs. Les blocages régionaux liés à l'absence de règlement israélo-arabe ont permis aux acteurs religieux de sortir Aïn el-Héloué de l'histoire palestinienne pour l'inscrire dans la géographie d'une oumma islamique humiliée par les forces impies de l'étranger. Autrefois pourvoyeur de fedayins au temps glorieux de la "révolution palestinienne ", le camp appartient désormais à l'espace imaginaire de l'islamisme radical, à la fois lieu de recrutement et point de chute pour militants traqués dans leur pays d'origine. Une telle métamorphose n'a pas fait disparaitre la Palestine, mais celleci a perdu son épaisseur historique en étant amalgamée à d'autres luttes, en Tchétchénie, en Afghanistan ou en Irak. Le contexte local de rupture avec la société libanaise, l'exposition continue à des thématiques de révolte religieuse dans les mosquées et les instituts d'enseignement islamique ont eu pour effet de favoriser la naissance de nouvelles formes d'identifications et de susciter chez certains un rejet d'un héritage familial encore marqué par le nationalisme palestinien. Plusieurs dizaines de volontaires ont ainsi quitté Aïn el-Héloué pour combattre les troupes américaines en Irak, à l'instar du fils de Jamâl sleïmân, mort dans une opération suicide dans la zone verte à Bagdad en 2004, sans que son père ait eu connaissance de ses intentions.

Les mêmes considérations de survie expliquent l'emprunt des cheikhs salafistes au discours du Hezbollah sur le rôle de la Résistance face aux menaces israéliennes. La diffusion dans les camps de réfugiés palestiniens d'un islamisme d'orientation salafiste a entrainé une exacerbation du clivage confessionnel entre chi'ites et sunnites exacerbation qui explique en partie l'impuissance de l'organisation libanaise à établir des mosquées ou des centres religieux dans les camps. Vu depuis les mosquées salafistes de Aïn el-Héloué ou de Nahr al-Bâred, le Hezbollah est une organisation hypocrite qui a verrouillé à son seul profit le front du Sud-Liban en parant sa résistance d'un label « islamique » alors qu'il s'agissait en réalité d'une résistance limitée aux seuls chi'ites libanais. Un tel comportement est venu confirmer a posteriori la duplicité chi'ite dénoncée par la tradition de l'hérésiographie sunnite. Mais cette exacerbation, largement entretenue à l'intérieur des cercles religieux, ne doit pas transparaître à l'extérieur, puisque rien n'est censé entraver l'expression d'une solidarité tactique avec le principal adversaire des États-Unis au Liban. Le conflit confessionnel s'efface alors devant les nécessités de la politique. La publication, à la fin du mois de juillet 2005, d'un document attribué à Abou Mas'ab al-Zarkaoui ${ }^{17}$ appelant au meurtre d'une série de personnalités chi'ites au Liban brouille cet effort tactique. Si son authenticité était établie, pareil document illustrerait les agendas croisés de deux catégories d'acteurs qui se réclament du même corpus idéologique, mais dont les situations respectives induisent des stratégies locales totalement opposées.

\section{Conclusion}

$35 \mathrm{Au}$ total, le dossier palestinien apparaît comme un indicateur du poids de l'influence syrienne au Liban ainsi qu'une illustration de la difficulté éprouvée par les différents acteurs, aussi bien Libanais que Palestiniens, à penser leur existence politique en dehors de cette influence. Paradoxalement, celle-ci a d'abord été intériorisée par ceux qui ont eu le plus à souffrir de la tutelle syrienne pendant ces quinze dernières années. Ce phénomène engage plusieurs types de causes et de responsabilités. Il résulte, pour partie, de la régression de l'analyse politique auprès des militants, ou, plus exactement, 
de la crise des instruments de socialisation politique auprès de la population palestinienne. Dans le climat de peur qui a prévalu pendant l'après-guerre libanais, les lieux de l'apprentissage politique et du débat ont progressivement disparu dans un milieu autrefois rompu aux délices de la conversation politique, pour faire place à une démagogie islamiste spécialisée dans l'accusation de trahison interne (takhwîn) face à toute posture critique. Une autre responsabilité remonte directement aux différents acteurs de la vie politique libanaise, qui, à dessein ou par ignorance, ont donné de l'enjeu palestinien au Liban une vision essentialiste et existentielle propre à l'entretien d'une dialectique de la peur exploitée à l'intérieur des camps par ceux qui voulaient conserver leurs positions de pouvoir ${ }^{18}$. Justifiée par la volonté de rendre les Palestiniens « inéligibles pour l'implantation », la politique du général Jamil al-Sayyid aura surtout eu pour effet de rendre la jeune génération « inéligible pour l'émigration ", en raison de la régression dramatique du niveau d'éducation provoquée par la multiplicité des mesures restrictives. Face à cette situation, une responsabilité politique incombe aux vainqueurs des élections législatives de l'été 2005 qui, en dépit d'une majorité politique écrasante de 72 députés sur 128, n'ont pas su - ou pas encore su - traduire en termes politiques les effets de leur victoire électorale. La nouvelle équipe dirigeante, formée autour du clan Hariri, n'a pas cherché à briser le monopole construit par la Syrie et ses alliés sur le dossier palestinien au Liban, ni voulu entraver la diplomatie déployée par le Hezbollah dans le milieu des camps. Le discours sur le tawtîn remplit ici sa fonction majeure : empêcher le gouvernement de définir une politique alternative et maintenir la question au cœur des préoccupations l'appareil sécuritaire.

Le même constat s'impose pour décrire la position d'attente de Mahmoud 'Abbas. Si elle permet de conserver l'illusion d'un consensus parmi les différents acteurs palestiniens, celle-ci ne peut pas dissimuler l'ampleur des divisions, politiques et identitaires, à l'intérieur des camps. Le dépassement des clivages de la guerre libanaise passera donc par la reconnaissance des clivages créés par l'après-guerre. En conséquence, l'Autorité palestinienne devra assumer l'existence d'ennemis au sein des camps, qu'il lui faudra combattre, sans doute avec l'appui du gouvernement libanais.

La mise en place de cette politique est aussi tributaire des relations à venir entre le gouvernement libanais et le Hezbollah. L'influence syrienne, on l'a vu, passe très largement pas le canal politique et idéologique du Hezbollah et de ses alliés dans le champ politique libanais. Même s'il demeure difficile de tirer des conclusions qui peuvent être démenties dans les prochains mois, en raison de l'extrême volatilité de la situation, la question du désarmement des camps - comme celle de son propre désarmement - montre que le Hezbollah continue de faire peser une hypothèque considérable sur la vie politique, en raison de sa capacité à dramatiser les enjeux en fonction de ses intérêts, tout en obtenant des garanties en termes de positions d'influence dans le système institutionnel. Ses dirigeants continuent de considérer qu'un redéploiement de l'armée libanaise dans le Sud-Liban, selon les propres termes de la résolution 1614 adoptée par le Conseil de sécurité le 29 juillet, transformerait cette institution en "gardienne des frontières israéliennes". Ces considérations rentrent également dans un cadre régional au sein duquel le Hezbollah se perçoit comme un élément dans un ensemble plus vaste dominé par le contentieux entre les États-Unis et l'Iran. Face à ce type de discours et de situation, les Palestiniens du Liban devront sortir des pièges d'une solidarité idéologique qui pourrait les replacer au cœur 
de contradictions libanaises et internationales qu'ils ne maîtrisent pas, et dont ils pourraient être à nouveau les victimes.

\section{NOTES}

1. Signés en octobre 1989 dans la ville saoudienne de Taëf pour sortir le pays de la guerre civile, intégrés dans le corpus constitutionnel en juillet 1990, les accords de Taëf ont transféré l'essentiel du pouvoir exécutif du président de la République, de confession maronite, au Conseil des ministres, placé sous l'autorité d'un premier ministre sunnite. Ils prévoyaient aussi un échéancier pour le « redéploiement » des troupes syriennes, ainsi que la dissolution de toutes les milices. Le texte a été détourné de sa vocation consensuelle par la pratique politique syrienne, qui en a subverti le sens et monopolisé l'interprétation, en rendant caduques les dispositions relatives au retrait de l'armée syrienne du Liban et à la dissolution de toutes les milices. Lorsque Bachar se vit confier par son père Hafez al-Assad la responsabilité du dossier libanais en 1998, il décida de faire de la présidence le principal canal de l'influence syrienne au Liban, en plaçant sa confiance dans l'autorité du général Émile Lahoud, nommé président de la République au mois de novembre de la même année.

2. En ce sens, la résolution 1559 ne s'inscrit pas dans la problématique néo-conservatrice du "changement de régime", mais cherche plutôt à empêcher le basculement définitif du Liban vers un ordre autoritaire, en appelant au respect des bases parlementaires du régime libanais.

3. Mis en place en 1949 par l'Assemblée générale des Nations unies et effective en mai 1950, l'UNRWA (United Relief and Works Agency, en français l'office de secours et de travaux des Nations unies pour les réfugiés de Palestine) est chargé d'apporter une aide aux réfugiés palestiniens qui ont été contraints de fuir leur foyer lors de la création de l'État d'Israël en 1948. Plus de 4 millions de réfugiés sont aujourd'hui enregistrés par l'UNRWA, dont la zone d'activité comprend les territoires palestiniens (952 295 pour Gaza et 682657 pour la Cisjordanie) et les pays arabes voisins (1 776669 pour la Jordanie, 421737 pour la Syrie et 399152 pour le Liban selon les chiffres fournis par le bureau d'information de l'UNRWA (avril 2005).

4. Les camps les plus importants sont répartis près des grandes villes du littoral : Aïn el-Héloué (44 775 réfugiés enregistrés) et Mié-Mié (4995), situés à l'est de Saïda au Sud, Nahr al-Bâred (28 931) et Baddâwi (15 982), dans la banlieue de Tripoli au Nord, Rachidiyyé (25 081) et Bourj alChémali (18 375), près de Tyr, et Bourj al-Barjneh $(20162)$, dans la banlieue sud de Beyrouth. Même si leurs noms restent désormais attachés à la mémoire des massacres de 1982, les camps de Sabra et Chatila se sont dissous dans leur environnement humain : Sabra a été détruit et Chatila s'apparente de plus en plus à un quartier populaire occupé par un lumpenprolétariat kurde, syrien et palestinien.

5. Pour une analyse de la situation socio-économique des Palestiniens au Liban après la guerre, voir Simon Haddad, The Palestinian Impasse in Lebanon. The Politics of Refugee Integration, Sussex Academic Press, 2003.

6. Dans son paragraphe 11, la résolution 194, votée le 11 décembre 1948 par l'Assemblée générale, dit expressément « qu'il y a lieu de permettre aux réfugiés qui le désirent de rentrer dans leurs foyers le plus tôt possible et de vivre en paix avec leurs voisins et que des indemnités doivent être payées à titre de compensation pour les biens de ceux qui décident de ne pas rentrer dans leurs foyers ». 
7. Pour un récit complet de l'histoire des réfugiés palestiniens au Liban, voir les deux ouvrages de Rosemary Sayigh, Palestinians : from Peasants to Revolutionaries (Londres, Zed Books, 1979) et Too Many Enemies : the Palestinian Experience in Lebanon (Londres, Zed Books, 1994).

8. L'ancien directeur général des affaires civiles palestiniennes au ministère de l'Intérieur de 1994 à 1998, Khalil Chatawi, reconnaît lui-même que « le dossier était traité en priorité par Jamil al-Sayyid et Émile Lahoud». Il lui était, par conséquent, interdit de nouer des relations avec les responsables du Fatah au Liban. Entretien avec l'auteur, Beyrouth, août 2005.

9. Entretien avec Fathi Abou al-'Urdât, secrétaire général du Fatah pour la région de Saïda et membre de la délégation palestinienne qui s'est rendue à Damas, juillet 2005, Saïda.

10. Entretien donné par Sultan Abou al-'Aynân à l'hebdomadaire libanais Al-Hawâdith, le 8 juillet 2005.

11. En 1994, le gouvernement libanais a naturalisé par décret 5600 familles issues de sept villages situés en territoire israélien, dans une zone frontalière revendiquée par le gouvernement libanais.

12. Prise par le ministre du Travail Trad Hamadé au sein du gouvernement sortant de Najib Miqati, la décision 1/79 élargit au bénéfice des Palestiniens les dispositions d'un décret du 18 septembre 1964 qui autorise certaines catégories d'étrangers à exercer des emplois limitativement énumérés par le texte administratif. Selon cette décision, les Palestiniens nés au Liban et enregistrés auprès du ministère de l'Intérieur pourront, moyennant l'achat d'un permis de travail, exercer des « petits métiers » (gardiens d'immeuble, chauffeur, mécanicien, cuisinier, etc.) ce qui ne leur était plus autorisé depuis la fin de la guerre. En revanche, il leur est toujours interdit d'avoir une activité commerciale ou d'exercer une profession libérale, c'est-à-dire une activité régie par un ordre professionnel. De même, ceux qui pourront travailler n'auront pas de protection sociale, puisque celle-ci n'est accordée aux étrangers que sous condition de réciprocité.

13. Après l'évacuation du Sud-Liban par l'armée israélienne en mai 2000, le Hezbollah a tiré prétexte du maintien de l'occupation israélienne sur les "fermes de Chebaa " - une bande territoriale situé à l'ouest du Mont-Hermon, aux confins de la frontière syro-libanaise - pour justifier la continuation d'une guérilla armée, conformément à la position du gouvernement libanais selon laquelle la résolution 425 sur l'obligation pour Israël d'évacuer le Sud-Liban n'a pas été entièrement appliquée. Pour le représentant du secrétaire général de l'ONU au contraire, le lieu-dit des fermes de Chebaa a été occupé par Israël lors du conflit de 1967 contre la Syrie. À ce titre, il sort du champ d'application de la 425 pour rentrer dans celui de la 242. Par ailleurs, l'ONU a dessiné une «ligne bleue » pour représenter la frontière israélo-libanaise, laquelle "ligne bleue » exclut les fermes de Chebaa du domaine libanais.

14. Sur le rôle des milieux jihâdistes dans les camps palestiniens au Liban, voir Bernard Rougier, Le jihad au quotidien, PUF, 2004. On se reportera également à l'article « Dynamique religieuse et identité nationale dans les camps de réfugiés palestiniens» dans la revue Maghreb-Machrek, $\mathrm{n}^{\circ} 176$, Été 2003.

15. Cassette de l'émission enregistrée dans les locaux de la mosquée al-Nour, Aïn el-Héloué, avril 2005.

16. Entretien dans le camp de Nahr al-Bâred, avril 2005.

17. Né le 30 octobre 1966 d'une famille pauvre de Zarqa dans la banlieue d'Amman, issu de l'importante tribu bédouine des Banu Hassan, le Jordanien Abou Mas'ab al-Zarqawi - de son vrai nom Ahmed Fadîl Nazâl al-Khalâylat - est, depuis 2004, le représentant du réseau al-Qaïda en Irak, au terme d'un parcours jihâdiste qui l'a mené de l'Afghanistan (1989) aux prisons jordaniennes (1996-1999) puis, de l'Afghanistan des Taliban (1999-2001) à l'Irak après l'intervention militaire américaine (2003). Depuis lors, son nom est associé aux actes de violence terroriste qui ont pris pour cible civils irakiens et otages occidentaux. 
18. Un effort pour briser cette dialectique est apparu parmi des intellectuels chrétiens, à l'exemple d'un ancien conseiller politique du parti Kata'eb, Joseph Abou Khalil, qui appelle l'État libanais à l'exercice de ses responsabilités politiques, en lui demandant notamment de coopérer avec l'Autorité palestinienne pour mettre fin à une politique de blocus social et politique qui apparaît comme un déni de gouvernance. Voir l'article de Joseph Abou Khalil: "Cette misère palestinienne n'est pas digne du Liban ", Al-Nahar, 26 octobre 2004.

\section{RÉSUMÉS}

À travers l'enjeu du désarmement des camps, cet article se propose de montrer comment le régime syrien et ses alliés libanais ont mobilisé des soutiens en milieu palestinien pour faire obstacle à l'application de la résolution 1559 des Nations Unies,, et la façon dont les acteurs politiques et religieux ont réagi à ces sollicitations. Entrée privilégiée pour étudier l'établissement d'un mode de gouvernement reposant essentiellement sur les services de sécurité syro-libanais depuis 1990, la question des camps témoigne aujourd'hui, depuis le retrait officiel de l'armée syrienne, d'une implication de plus en plus lourde du Hezbollah dans la gestion du dossier palestinien au Liban.

This paper analyses how the Syrian regime and its Lebanese allies have mobilized support from the Palestinian camps in Lebanon to impede the application of UN Security Council Resolution 1559 ; and how political and religious forces have reacted to this strategy. In this regard, the Palestinian camps in Lebanon offer a particular opportunity to perceive how, since the official withdrawal of the Syrian army, Hezbollah has become increasingly involved in the Palestinian issue in Lebanon.

\section{INDEX}

Keywords : Lebanon, Syria, Palestinian camps, Hezbollah, UN

Index géographique : Liban, Syrie

Mots-clés : camps palestiniens, Hezbollah, ONU

\section{AUTEUR}

\section{BERNARD ROUGIER}

Chercheur à l'Institut français du Proche-Orient (IFPO), Amman. Chercheur à l'Institut français du Proche-Orient (IFPO), Amman.

Diplômé de l'Institut d'études politiques de Paris, docteur en sciences politiques et arabisant, Bernard Rougier est chercheur à l'Institut français du Proche-Orient (IFPO) à Amman, en Jordanie. Il travaille sur les différentes expressions de l'islamisme dans l'Orient arabe. Il est l'auteur d'un ouvrage sur le rôle des réseaux jihadistes dans les camps palestiniens du Liban Le jihad au quotidien (Paris, PUF, 2004), dont la version 
anglaise sera prochainement publiée chez Harvard University Press.

brougier@hotmail.com 